\title{
Research Article \\ The Application of Memetic Algorithms for Forearm Crutch Design: A Case Study
}

\author{
Teresa Wu, ${ }^{1}$ Som Soni, ${ }^{2}$ Mengqi Hu, ${ }^{1}$ Fan Li, ${ }^{1}$ and Adedeji Badiru ${ }^{3}$ \\ ${ }^{1}$ Industrial Engineering Program, School of Computing, Informatics, Decision Systems Engineering, \\ Arizona State University, Tempe, AZ 85287, USA \\ ${ }^{2}$ Center for Rapid Product Development, Air Force Institute of Technology, \\ Wright-Patterson Air Force Base, Dayton, OH 45433-7765, USA \\ ${ }^{3}$ Department of Systems and Engineering Management, Air Force Institute of Technology, \\ Wright-Patterson Air Force Base, Dayton, OH 45433-7765, USA \\ Correspondence should be addressed to Teresa Wu, teresa.wu@asu.edu
}

Received 31 July 2010; Revised 16 December 2010; Accepted 5 January 2011

Academic Editor: Reza Jazar

Copyright (C) 2011 Teresa Wu et al. This is an open access article distributed under the Creative Commons Attribution License, which permits unrestricted use, distribution, and reproduction in any medium, provided the original work is properly cited.

Product design has normally been performed by teams, each with expertise in a specific discipline such as material, structural, and electrical systems. Traditionally, each team would use its member's experience and knowledge to develop the design sequentially. Collaborative design decisions explore the use of optimization methods to solve the design problem incorporating a number of disciplines simultaneously. It is known that such optimized product design is superior to the design found by optimizing each discipline sequentially due to the fact that it enables the exploitation of the interactions between the disciplines. In this paper, a bi-level decentralized framework based on Memetic Algorithm (MA) is proposed for collaborative design decision making using forearm crutch as the case. Two major decisions are considered: the weight and the strength. We introduce two design agents for each of the decisions. At the system level, one additional agent termed facilitator agent is created. Its main function is to locate the optimal solution for the system objective function which is derived from the Pareto concept. Thus to Pareto optimum for both weight and strength is obtained. It is demonstrated that the proposed model can converge to Pareto solutions.

\section{Introduction}

Under collaborative design paradigm, the first common topic is Multidisciplinary Design Optimization (MDO) which is defined as "an area of research concerned with developing systematic approaches to the design of complex engineering artifacts and systems governed by interacting physical phenomena" [1]. Researchers agree that interdisciplinary coupling in the engineering systems presents challenges in formulating and solving the MDO problems. 
The interaction between design analysis and optimization modules and multitudes of users is complicated by departmental and organizational divisions. According to Braun and Kroo [2], there are numerous design problems where the product is so complex that a coupled analysis driven by a single design optimizer is not practical. This is because either the lead time needed to integrate the analysis or the lag introduced by disciplinary sequencing is too long. Some researchers have taken project management as a means to facilitate and coordinate the design among multiple disciplines [3,4].

Early advances in MDO involve problem formulations that circumvent the organizational challenges, one of which is to protect disciplinary privacy by not sharing full information among the disciplines. It is assumed that a single analyst has complete knowledge of all the disciplines. As indicated by Sobiesczanaki-Sobieski and Haftka [5], most of the work at this phase aims to tackle the problems by a single group of designers within one single enterprise environment where the group of designers share a common goal and require less disciplinary optimum. The next phase of MDO gives birth to two major techniques: optimization by linear decomposition (OLD) and collaborative optimization (CO). These techniques involve decomposition along disciplinary lines and global sensitivity methods that undertake overall system optimization with minimal changes to disciplinary design and analysis. However, Alexandrov and Lewis [6] explore the analytical and computational properties of these techniques and conclude that disciplinary autonomy often causes computational and analytical difficulties which result in severe convergence problems.

Parallel to these MDO developments, there also evolves the field of decision-based design [7-12] which provides a means to model the decisions encountered in design and aims at finding "satisfying" solutions $[13,14]$. Research in Decision-Based Design includes the use of adaptive programming in design optimization [15], the use of discrete choice analysis for demand modeling $[10,11]$. In addition, there has been extensive research ranging from single-objective Decision-Based Design [10] to multiobjective models [16, 17]. It combines game theory, utility theory, and decision science for collaborative design which can be conducted among a group of designers from different enterprises. This technique has several computational difficulties in calculating the "best reply correspondence" and the rational reaction sets especially when the designs are very complex. Besides, several approximations like using response surfaces within these techniques make them prone to errors [18].

Note that most of the methods reviewed above have strict assumption on the utility functions and/or constraints (e.g., convexity and quasilinear of the functions) which limits the application to product design. In this research, we explore the use of a metaheuristic method, Memetic Algorithm (MA), and a combination of Local Search (LS) and Genetic Algorithm (GA) to forearm crutch design which has nonconvex objective for one of the decisions. Forearm crutches have been exclusively used by people with permanent disability. Nowadays, it is beginning to serve for some shorter-term purposes as well. The design of forearm crutch needs to consider multidisciplinary decisions. For example, the structure designer wants to ensure the design is lightweight. The material engineer wants composite material to have the right number of layers at right angles to make the product durable. The outsourcing engineer wants the supplier to provide low cost, high reliable, and light weight parts. Another important factor impacting the design is cost. Here, we introduce the design agent for each disciplinary decision problem and one system agent facilitating the communication among the design agents and guiding the design to converge. To achieve this, the overall decision space is partitioned into two sets: one for coupled variables (the ones shared by at least two designers) one for local variables (the ones that can be fully controlled by each designer). Next, an iterative process between design agent decisions 
on local variables and facilitator agent decisions on the whole design space launches. It is demonstrated that a converged Pareto optimum is achieved after a number of iterations for the forearm crutch design which has nonlinear form decision functions.

This paper is organized as follows: the related literature is briefly reviewed in Section 2, followed by the detailed explanation on the proposed bi-level decentralized framework in Section 3. The forearm crutch case is explained in Section 4 with the conclusions being drawn in Section 5 .

\section{Literature Review}

Collaborative Optimization (CO), introduced by Braun and Kroo [2], is a bi-level optimization approach where a complex problem is hierarchically decomposed along disciplinary boundaries into a number of subproblems which are brought into multidisciplinary agreement by a system level coordination process. With the use of local subspace optimizers each discipline is given complete control over its local design variables subject to its own disciplinary constraints. The system level problem sets up target values for variables from each discipline. Each discipline sets the objectives to minimize the discrepancy between the disciplinary variable values and the target values. The system-level optimization problem is formulated as minimizing a global objective subject to interdisciplinary consistency constraints. The interdisciplinary consistency constraints are equality constraints that match the system-level variables with the disciplinary variables. In Optimization by Linear Decomposition (OLD) [20-22], the disciplines are given the autonomous task of minimizing disciplinary design infeasibility while maintaining system-level consistency. The system-level problem is to drive design infeasibility to zero. At the local-level problem the disciplines use their local degrees of freedom to minimize the violation of the disciplinary design constraints, subject to matching the target value for the disciplinary output that is fed into the discipline. Balling et al. [23] introduce a combination of CO and OLD where the disciplinary subproblems minimize the discrepancy in the system-level targets as well as the disciplinary design infeasibility given the disciplinary design constraints.

Both CO and OLD depend on a design problem's amenability to hierarchical decomposition with the system objective explicitly defined. On the other hand, concurrent Subspace optimization (CSSO) [21] is a nonhierarchic system optimization algorithm that optimizes decomposed subspaces concurrently, followed by a coordination procedure for directing system problem convergence and resolving subspace conflicts. In CSSO, each subspace optimization is a system level problem with respect to the subset of the total system design variables. Within the subspace optimization, the nonlocal variables that are required to evaluate the objective and the constraint functions are approximated using global sensitivity equations (GSE). Interested readers please refer to [21], for detailed description of GSEs.

The bi-level integrated synthesis (BLISS) [24] method uses a gradient-guided path to reach the improved system design, alternating between the set of modular design spaces (the disciplinary problems) and the system level design space. BLISS is an all-in-one-like method in that the complete system analysis is performed to maintain multidisciplinary feasibility at the beginning of each cycle of the path. The overall problem is decomposed such that a set of local optimization problems deal with the detailed local variables which are large in number and one system level optimization problem deals with a small number of global variables.

Decision-based design [9-11,25] is a paradigm focusing on distributed and collaborative design efforts. For the cases where continuous variables are used, adaptive linear programming [17] is employed; in case of mixed discrete and continuous variables, 


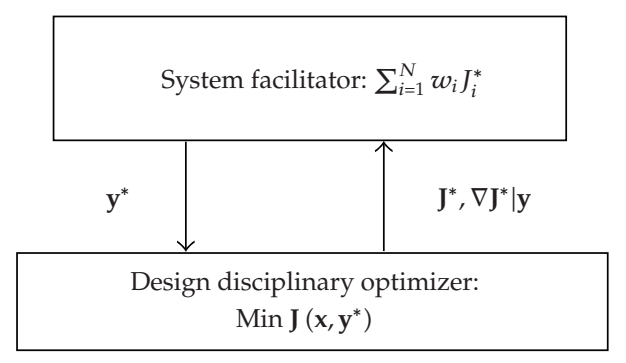

Figure 1: Overall decision framework.

Foraging-directed adaptive linear programming has been used [17]. In a noncooperative environment, game theoretic principles are used to arrive at the best overall design $[16,17]$. Recently, Design-for-Market system grows out of the decision-based Design and emerges as an area focusing on establishing a solid basis in decision theory, by taking microeconomics into account, to support engineering design. Kumar et al. [26] propose a hierarchical choice model based on discrete choice analysis to manage and analyze customer preference data in setting design targets. Azarm's group studies new product designs that are robust from two perspectives-from the engineering perspective in terms of accounting for uncertain parameters and from the market perspective in terms of accounting for variability in customer preferences measurement [27]. They conclude incorporating consumer heterogeneity in considering the variability in customer preferences may have significant impact on the ultimate design. Research led by Michalek explores the use of gametheoretic approach to finding market equilibrium under various regulation scenarios [28]. A metric for agility measurement is introduced by Seiger et al. [29] to explore the product development for mass customization.

In general, some common criticisms and/or challenges facing collaborative design decisions are the convergence and information sharing issues:

(i) Will the decision model converge? If yes, under what condition (assumptions on the function form and design spaces) will it converge? How fast will it converge?

(ii) Most models (CO, OLD, BLISS, etc.) take a top-down approach with the full knowledge of the design space (e.g., the form of utility functions, constraints) being available. For the cases when the design information is partially known, what decision model is appropriate?

To address these challenges, we propose a general decision framework based on MA that allows distributed design teams to arrive at Pareto solutions which is in detail explained in the next section.

\section{Memetic Algorithm and Its Application to Collaborative Design}

Memetic algorithm (MA) is one of the emerging areas in evolutionary computation. It integrates genetic algorithm (GA) with local search (LS) to improve the efficiency of searching complex spaces. In MA, GA is used for global exploration while LS is employed for local exploitation. The complementary nature of GA and LS makes MA an attractive approach for large-scale, complex problems, for example, collaborative design. 


\subsection{Proposed Framework for Collaborative Design}

Let us consider a general collaborative design with $N$ design teams. The problem can be represented as

$$
\begin{aligned}
\text { Min } & \mathbf{J}(\mathbf{x}, \mathbf{y}) \\
\text { St. } & \mathbf{g}(\mathbf{x}) \leq 0, \\
& \mathbf{h}(\mathbf{x}, \mathbf{y}) \leq 0, \\
& x_{i}^{L B} \leq x_{i} \leq x_{i}^{U B}, \quad\left(i=1, \ldots, n_{1}\right), \\
& y_{i}^{L B} \leq y_{i} \leq y_{i}^{U B}, \quad\left(i=1, \ldots, n_{2}\right),
\end{aligned}
$$

where $\mathbf{J}=\left[J_{1}(\mathbf{x}, \mathbf{y}) \cdots J_{N}(\mathbf{x}, \mathbf{y})\right]^{T}, \mathbf{x}=\left[x_{1} \cdots x_{n 1}\right]^{T}, \mathbf{y}=\left[y_{1} \cdots y_{n 2}\right]^{T}, \mathbf{g}=\left[g_{1}(\mathbf{x}) \cdots g_{m 1}(\mathbf{x})\right]^{T}$, $\mathbf{h}=\left[h_{1}(\mathbf{x}, \mathbf{y}) \cdots h_{m 2}(\mathbf{x}, \mathbf{y})\right]^{T}, \mathbf{x}$ is the set of $n_{1}$ local variables, $\mathbf{y}$ is the set of $n_{2}$ coupled variables, $\mathbf{g}$ is the set of $m_{1}$ local constraints, and $\mathbf{h}$ is the set of $m_{2}$ coupled constraints.

Figure 1 illustrates the iterative decision process between system facilitator agent and disciplinary design agents. First, the facilitator initializes the global solution space over both local and coupled variables. For any solution, for example, $\left[\mathbf{x}^{*}, \mathbf{y}^{*}\right]$, each design agent will execute local optimizer over the subdesign space which consists of $\mathbf{x}$ only, that is $\operatorname{Min} \mathbf{J}\left(\mathbf{x}, \mathbf{y}^{*}\right)$ (given $\mathbf{y}=\mathbf{y}^{*}$ ). The results fed back to the facilitator are the values of objective function and the gradient of objective function over coupled variables. The facilitator will employ local search for the recent results updated by each designer using the related gradient information for the improved design; next, traditional GA operators, crossover, and mutation are applied to introduce new candidates to the solution space.

\subsection{Pseudocode}

Parameters

$N$ : no. of disciplinary design agents;

$w_{i}$ : weight for the objective function of $i$ th disciplinary design agent, where $i=$ $1, \ldots, N$;

$1 / W$ : weight step size;

$P$ : population size;

As shown in Pseudocode, there exist three loops, from outer to inner in the proposed method: weight enumeration (lines 11-37), GA loop (lines 13-37), and local search loop (lines 24-28). That is, given a weights combination (e.g., $w_{1}=0.3, w_{2}=0.7$ for two agents), GA is triggered which applies crossover and mutation operators and selection mechanism (in this case study, elitism selection is employed) for the population update. In addition, for the updated population, local search is further employed to identify improved solutions within the neighborhood. This is achieved by having subgradient information from each designer on the coupled variables fed back to the facilitator. Specifically, given any chromosome from the population, each design agent assumes the coupled variables are set and thus conducts optimization on the local variables only. Each design agents would also 


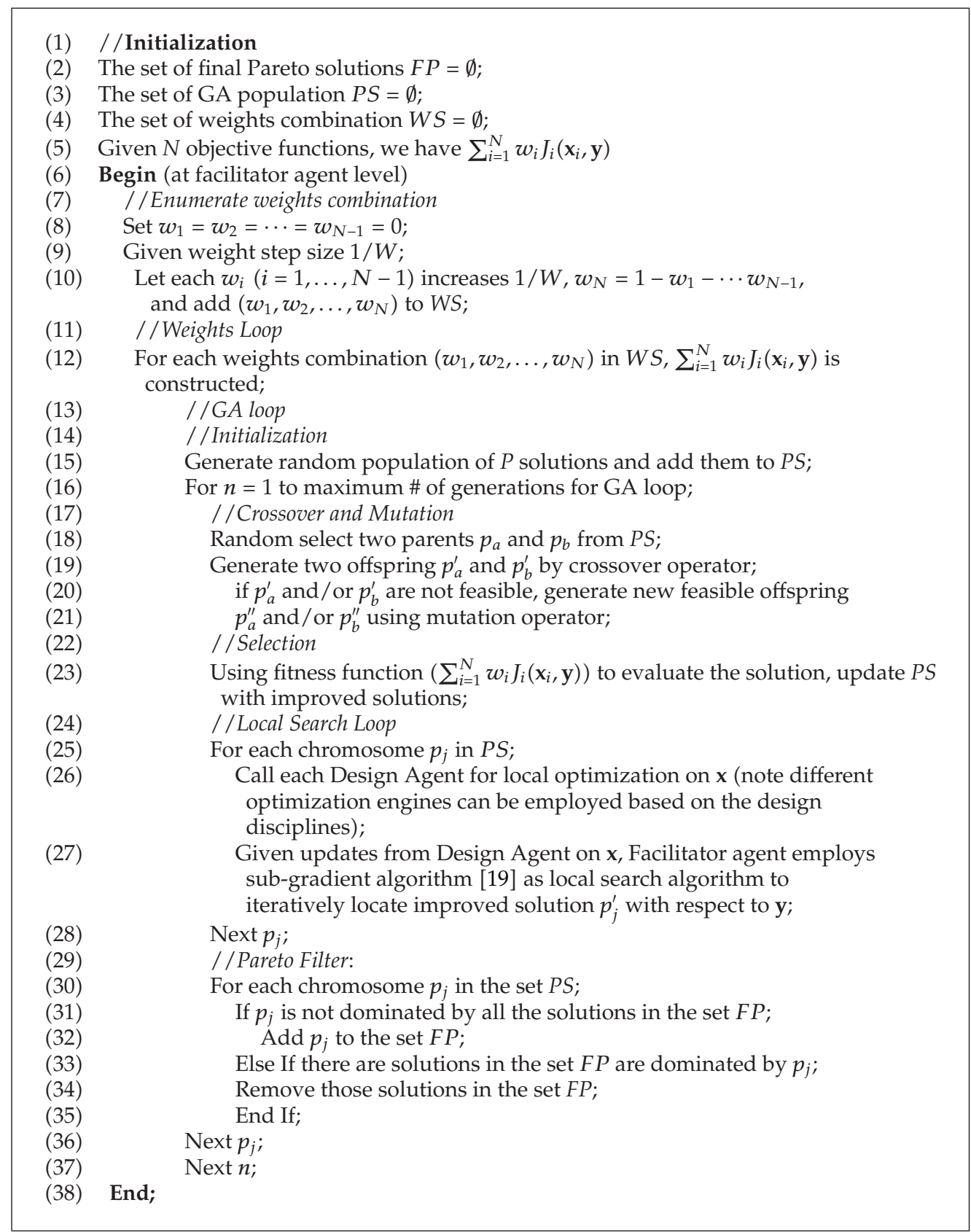

\section{PSEUdOCODE 1}

study the gradients on the coupled variables. Thus, given the values of the coupled variables, both the optimal design on local variables and the subgradient on the coupled variables are sent back to the facilitator. Since the priorities of the objective functions reflected by the weight assignments are enumerated exhaustively, all the possible Pareto solutions are located forming the Pareto frontier. In some cases where the priority is known, the weight loop can be removed. Please note that the Pareto filter operation (lines 29-36) is triggered by the facilitator 


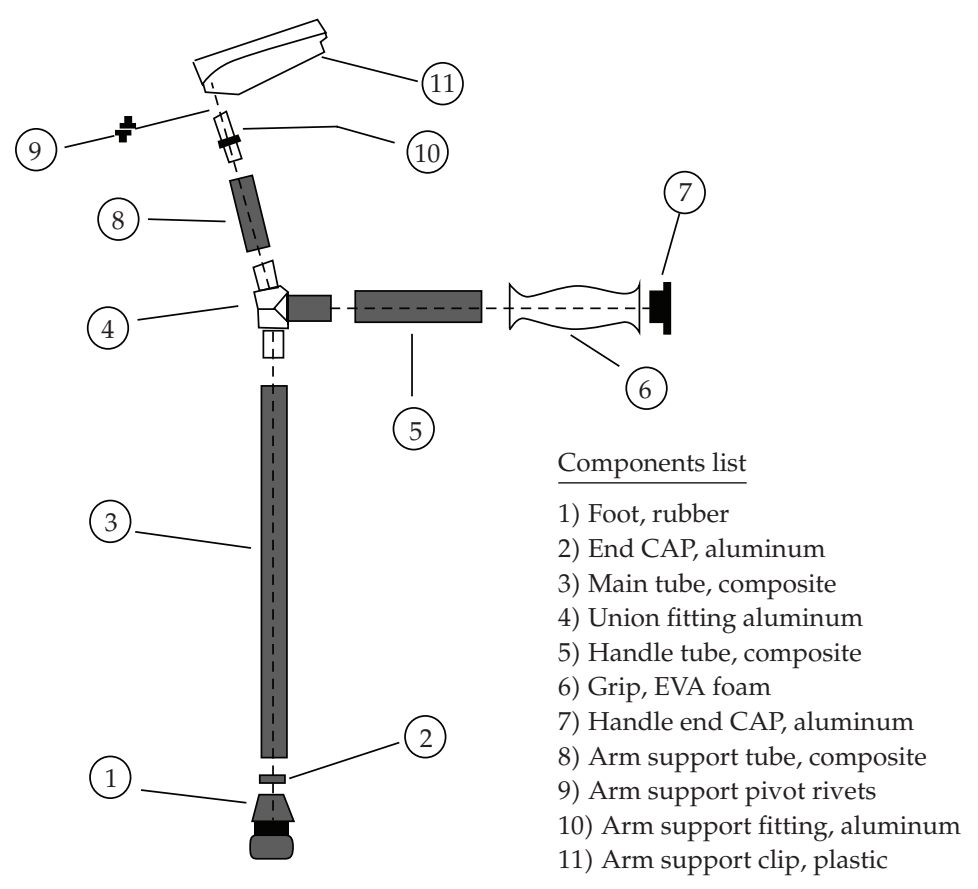

Figure 2: Exploded view of a forearm crutch.

within each weight combination. That is, it is possible that some Pareto solutions given a specific weight may be dominated by the Pareto solutions obtained with other weights.

One distinguishable feature of this proposed approach from other existing methods is that the information exchanged iteratively between the facilitator and the design agent is point values instead of function forms. For example, passing from the facilitator to the design agent (top-down) is the values of the coupled variable; passing from the design agent back to the facilitator (bottom-up) is the values of the objective function and associated gradient values. Thus, one main advantage of this approach is that a "black box" disciplinary optimizer can be easily plugged in. Secondly, the facilitator explores the solution space based on the solution candidates $\left(\mathbf{x}^{*}, \mathbf{y}^{*}\right)$ and the performance candidates $\left(\mathbf{J}^{*}\right)$ only without knowing the function formulation. This enables a truly decentralized decision to be reached in that no full knowledge of the design space is required.

In the next section, an industry case is explored to demonstrate the applicability of the proposed framework.

\section{Forearm Crutch Design}

Crutches are mobility aids used to counter mobility impairment or an injury that limits walking ability. Forearm crutches are used by slipping the arm into a cuff and holding the grip (Figure 2). It has been increasingly used for patients with shorter-term needs. Earlier study conducted by National Medical Expenditure Survey (NMHS) in 1987 indicates that an estimated 9.5 million (4\%) noninstitutionalized US civilians experience difficulty in performing basic life activities; some need crutches for leg support for walking. This number increases due to the baby boomer effect. 
Typical forearm crutches are made of aluminum and are criticized by customers for being heavy, noisy, and less durable. Customers suggest that a small reduction in the weight of forearm crutches would significantly reduce the fatigue experienced by crutch users. However, the reduction in weight should not be accompanied by a strength reduction. Most crutches on the market are designed for temporary use and wear out quickly. Crutch users commonly have to replace their crutches two to three times a year. This drives the need to redesign forearm crutches which are robust, appropriate for a wide range of users from lighter weight adults to users weighing up to 250 pounds with considerable upper body strength and who may use them aggressively on a continuous basis.

One solution is to use composite material for crutch which is light weight with good performance in strength. However, it comes with relatively expensive cost. After indepth marketing survey, the design team decides to outsource the aluminum union fitting (component no. 4 in Figure 2), use appropriate composite tube and apply adhesive from Hysol to bond the tubes with union fitting.

\section{Aluminum Union}

The design team first develops a computer model based on finite element method to determine the necessary wall thickness and to calculate the load on the handle necessary to produce yielding. An aluminum union which costs $\$ 150$ and stands $\geq 630 \mathrm{lbs}$ is used. The use of Hysol adhesive to bond the union with the tube needs to be tested to ensure that the strength requirement is satisfied.

\section{Composite Tube}

A typical composite tube is $39^{\prime \prime}$ in length. The tube can be cut into smaller pieces for the forearm crutch assembly. Approximately 2.5 tubes are needed to make a pair of crutches. Here, three smaller tubes are used as: handle (component no. 5 in Figure 2) which is fixed as 4.75 inch, arm support tube (component no. 8 in Figure 2) which usually ranges from 6.5 to 7.8 inch, and main tube (component no. 3 in Figure 2) which ranges from 30.6875 to 34.25 inch. The inner diameter of the tube is critical to maintain the proper bondline thickness for each adhesive joint. It ranges from 0.7605 to $0.7735 \mathrm{inch}$. The outer diameter is determined by the number of plies, and it ranges from 0.922 to 0.935 inch. Usually, the arm support tube is less concerned with strength, the main tube needs to be tested for the strength consideration.

Thus, we have two decision problems constructed: weight and strength.

\subsection{Design Problem Formulation}

\subsubsection{Design Agent for Weight Decision}

In this research, we focus on the weights of the tubes (arm support tube-component no. 8 and main tube-component no. 3 ) and a minimization problem is introduced as:

$$
\begin{array}{ll}
\text { Min: } & W=W_{u}+W_{L \prime} \\
\text { St: } & W_{u}=\rho \pi\left[\left(\frac{D_{o}}{2}\right)^{2}-\left(\frac{D_{i}}{2}\right)^{2}\right] \times L_{u},
\end{array}
$$




$$
\begin{aligned}
& W_{L}=\rho \pi\left[\left(\frac{D_{o}}{2}\right)^{2}-\left(\frac{D_{i}}{2}\right)^{2}\right] \times L_{L}, \\
& 30.6875 \leq L_{L} \leq 34.25, \\
& 6.5 \leq L_{u} \leq 7.8 \\
& 0.922 \leq D_{o} \leq 0.935, \\
& 0.7605 \leq D_{i}+\frac{2 T}{1000} \leq 0.7735,
\end{aligned}
$$

where $W_{u}(\mathrm{lb})$ is the weight of arm support tube, $W_{L}(\mathrm{lb})$ is the weight of main tube, $\rho$ $\left(\mathrm{Lb} /\right.$ inch $\left.^{3}\right)$ is the density of the composite tube, which is 0.08 in this paper, $L_{u}$ (inch) is the length of the arm support tube, $L_{L}$ (inch) is the length of the main tube, $D_{o}$ (inch) is the outer diameter, $D_{i}$ (inch) is the inner diameter and $T$ (mils, 1 mils $=0.001 \mathrm{inch}$ ) is the bondline adhesive material thickness.

\subsubsection{Design Agent for Strength Decision}

Since the strength from aluminum fitting is satisfactory from finite element analysis (FEM), the strength model will consider two potential failures: the adhesive applied joint, and the strength of the main tube. Thus, the problem is constructed as:

$$
\begin{array}{ll}
\text { Max: } & S=\operatorname{Min}\left(S_{L}, S_{A}\right), \\
\text { St: } & S_{L}=\frac{\pi E I}{L_{L}^{2}}, \\
& I=\frac{\pi\left(D_{o}^{4}-D_{i}^{4}\right)}{64}, \\
& 12 \leq E \leq 16, \\
& S_{A}=\left(-6.0386 T^{2}+7.7811 T+4644.5\right) \times \frac{\pi}{4} \times\left(D_{o}^{2}-D_{i}^{2}\right), \\
& 0.922 \leq D_{o} \leq 0.935, \\
& 0.7605 \leq D_{i}+\frac{2 T}{1000} \leq 0.7735, \\
& 30.6875 \leq L_{L} \leq 34.25, \\
& 0 \leq T \leq 17,
\end{array}
$$

where $S_{L}(\mathrm{lbs})$ is the strength of the bottom of the lower tube, $E\left(\mathrm{msi}, 1 \mathrm{msi}=10^{6} \mathrm{psi}\right.$ ) is the modulus of elasticity, $I\left(\right.$ inch $\left.^{4}\right)$ is the area moment of inertia and $S_{A}$ (lbs) is the strength of the joint after applying adhesive. 


\subsection{Implementation}

For the decision problems explained above, optimization code written in Matlab is executed. Here, we provide detailed explanation how the system problem is constructed and how the facilitator agent guides the design agents to converge to the solution using MA.

Step 1 (initialization). Given $w_{1}, w_{2}$, construct system search space as $w_{1} W^{*}-w_{2} S^{*}\left(W^{*}\right.$ and $S^{*}$ are the values of the objectives from each design agent, $w_{1}+w_{2}=1$ ).

Step 2 (real code genetic algorithm). The chromosome is represented with real numbers, that is $\left(L_{u}, L_{L}, D_{o}, D_{i}, T, E\right)$. Note that $L_{L}, D_{o}, D_{i}, T$ are coupled variables, $L_{u}$ is the local variable for weight agent and $E$ is the local variable for strength agent.

Substep 2.1 (initial population). For $\left(L_{u}, L_{L}, D_{o}, D_{i}, T, E\right)$, without losing of generalization, let assume that $a$ and $b$ represent the lower bound and upper bound of one of the variables and let $r$ be a random number $r \in[0,1]$, we get $(b-a) r+a$. Thus, a new chromosome is generated as for the initial population. A pool of 40 chromosomes is created.

Substep 2.2 (selection of parents). To ensure all chromosomes have the chances to be selected, solutions are classified into three groups according to their fitness: high fitness level, medium fitness level and low fitness level. The fitness is assessed based on $w_{1} W^{*}-w_{2} S^{*}$, the lower, the better.

Substep 2.3 (crossover). Given two chromosome $C_{1}=\left(L_{u^{\prime}}^{1} L_{L^{\prime}}^{1} D_{o^{\prime}}^{1} D_{i}^{1}, T^{1}, E^{1}\right)$ and $C_{2}=$ $\left(L_{u}^{2}, L_{L}^{2}, D_{o}^{2}, D_{i}^{2}, T^{2}, E^{2}\right)$, the offspring are generated as:

$$
\begin{aligned}
& C_{1}^{\prime}=\theta C_{1}+(1-\theta) C_{2} \\
& C_{2}^{\prime}=(1-\theta) C_{1}+\theta C_{2}
\end{aligned}
$$

where $\theta \in[0,1]$.

Substep 2.4 (mutation). Mutation is applied by simply generating a new feasible solution to replace the infeasible one.

Step 3 (local search). The facilitator agent applies subgradient method-based LS over coupled variables to improve the solutions. First, each design agent evaluates the gradients of the design decision problems (disciplinary) w.r.t the coupled variables. For example, given the coupled variables $L_{L}=L_{L}^{*}, D_{o}=D_{o}^{*}, D_{i}=D_{i}^{*}, T=T^{*}$, each decision problem is solved independently for $W^{*}$ and $S^{*}$. The gradients are obtained as

$$
\begin{aligned}
\lambda_{W, L_{L}}=\left.\frac{\partial W}{\partial L_{L}}\right|_{L_{L}=L_{L}^{*}, D_{o}=D_{o}^{*}, D_{i}=D_{i}^{*}, T=T^{*}}, & \lambda_{S, L_{L}}=\left.\frac{\partial S}{\partial L_{L}}\right|_{L_{L}=L_{L}^{*}, D_{o}=D_{o}^{*}, D_{i}=D_{i}^{*}, T=T^{*}}, \\
\lambda_{W, D_{o}}=\left.\frac{\partial W}{\partial D_{o}}\right|_{L_{L}=L_{L}^{*}, D_{o}=D_{o}^{*}, D_{i}=D_{i}^{*}, T=T^{*}}, & \lambda_{S, D_{o}}=\left.\frac{\partial S}{\partial D_{O}}\right|_{L_{L}=L_{L}^{*}, D_{o}=D_{o}^{*}, D_{i}=D_{i}^{*}, T=T^{*}}, \\
\lambda_{W, D_{i}}=\left.\frac{\partial W}{\partial D_{i}}\right|_{L_{L}=L_{L}^{*}, D_{o}=D_{o}^{*}, D_{i}=D_{i}^{*}, T=T^{*}}, & \lambda_{S, D_{i}}=\left.\frac{\partial S}{\partial D_{i}}\right|_{L_{L}=L_{L}^{*}, D_{o}=D_{o}^{*}, D_{i}=D_{i}^{*}, T=T^{*}}, \\
\lambda_{W, T}=\left.\frac{\partial W}{\partial T}\right|_{L_{L}=L_{L}^{*}, D_{o}=D_{o}^{*}, D_{i}=D_{i}^{*}, T=T^{*}}, & \lambda_{S, T}=\left.\frac{\partial S}{\partial T}\right|_{L_{L}=L_{L}^{*}, D_{o}=D_{o}^{*}, D_{i}=D_{i}^{*}, T=T^{*}} .
\end{aligned}
$$


The gradients of the system problem are then calculated as

$$
\begin{aligned}
& \lambda_{L_{L}}=w_{1} \lambda_{W, L_{L}}-w_{2} \lambda_{S, L_{L}}, \\
& \lambda_{D_{o}}=w_{1} \lambda_{W, D_{o}}-w_{2} \lambda_{S, D_{o}}, \\
& \lambda_{D_{i}}=w_{1} \lambda_{W, D_{i}}-w_{2} \lambda_{S, D_{i}}, \\
& \lambda_{T}=w_{1} \lambda_{W, T}-w_{2} \lambda_{S, T},
\end{aligned}
$$

based on $\lambda=\left[\lambda_{L_{L}}, \lambda_{D_{o}}, \lambda_{D_{i}}, \lambda_{T}\right]$ the facilitator agent employs nonsummable diminishing method to update the coupled variables. That is, at iteration $k+1$,

$$
\left[\begin{array}{c}
L_{L} \\
D_{o} \\
D_{i} \\
T
\end{array}\right]^{(k+1)}=\left[\begin{array}{c}
L_{L} \\
D_{o} \\
D_{i} \\
T
\end{array}\right]^{(k)}-\alpha_{k+1}\left[\begin{array}{c}
\lambda_{L_{L}} \\
\lambda_{D_{o}} \\
\lambda_{D_{i}} \\
\lambda_{T}
\end{array}\right]^{(k)},
$$

where step size $\alpha_{k}$ satisfies:

$$
\begin{aligned}
& \lim _{k \rightarrow \infty} \alpha_{k}=0, \\
& \sum_{k=1}^{\infty} \alpha_{k}=\infty .
\end{aligned}
$$

The coupled variables are updated based on the above subgradient method until no further improvement of the weighted system problem is required.

\subsection{Results and Analysis}

The Pareto frontier obtained by the proposed decentralized framework is shown in Figure 3. Please note the problem has Min-Max structure. Since this project focuses on the composite tube design (arm support tube-component no. 8 and main tube-component no. 3), the weight for the handle tube (component no. 5) is computed as

$$
\rho \pi\left[\left(\frac{D_{o}}{2}\right)^{2}-\left(\frac{D_{i}}{2}\right)^{2}\right] \times 4.75 .
$$

Other components in Figure 2 are outsourced with the weights summarized in Table 1.

We choose two Pareto solutions (A and B) to compare with the composite crutch from Ergonomics and the Invacare crutch which are two commercial products in Table 2. Apparently, most composite crutches outperform Ergonomics and Invacare for both weight and strength except Design B which outweighs Ergonomics by $0.0945 \mathrm{lb}$. However, Design B is much durable with strength being $1107 \mathrm{lbs}$ comparing to $715 \mathrm{lbs}$ of Ergonomics. 
Table 1: Weights for each component of the crutch.

\begin{tabular}{lc}
\hline Components (Figure 2) & Weight $(\mathrm{lb})$ \\
\hline$\# 2$ & 0.006 \\
$\# 4$ & 0.05 \\
$\# 7$ & 0.0074 \\
$\# 10$ & 0.025 \\
Others (\#1,\#6, \#9, \#11) & 0.2 \\
\hline
\end{tabular}

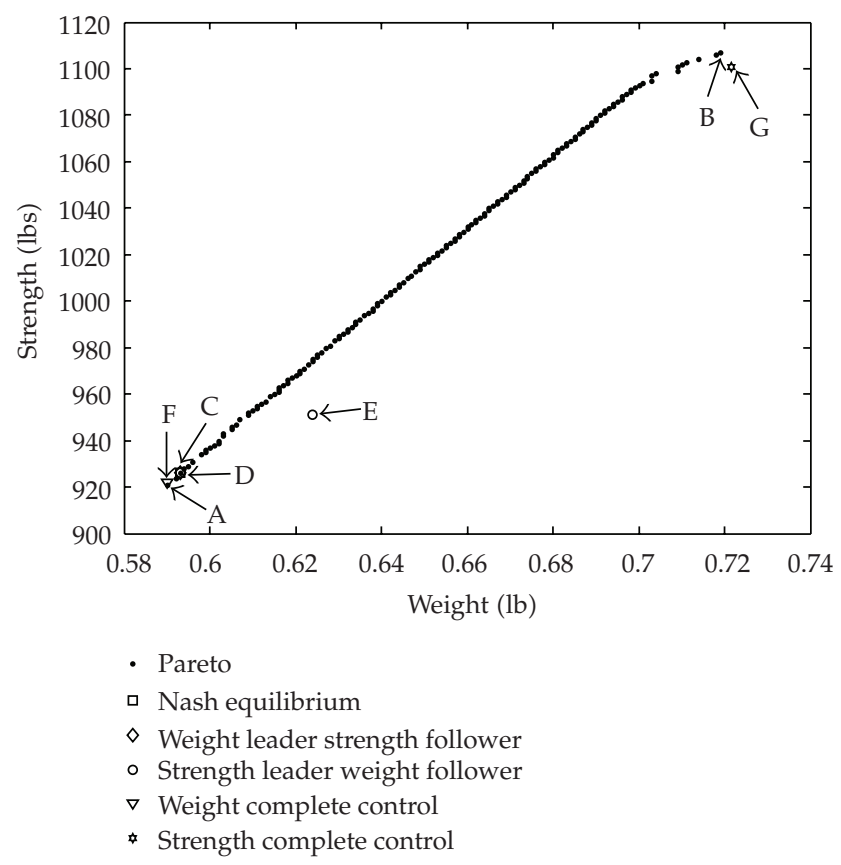

Figure 3: Pareto frontier in performance space for the crutch design.

Furthermore, game theoretical approaches including Nash equilibrium, Leader/ Follower games and Complete Control games are applied to the same problem for comparison study. In the game approaches, the weight design agent will control design variables $L_{u}, L_{L}, D_{o}$ and $D_{i}$, and design variables $T$ and $E$ are controlled by strength design agent. All the game theoretical solutions are demonstrated in Figure 3. As shown in Figure 3, solutions generally obtained from game theoretical approaches are not Pareto optimal (only the Nash equilibrium solution $C$ and one solution $D$ from the Leader/Follower game are on the frontier).

It is expected that the cost of the composite crutch will be high. In this case, it is around $\$ 460$ in total (tube and other components shown in Figure 2). The crutch produced by Invacare and Ergonomics price range is $\$ 60$ to $\$ 250$. Although the composite crutch is several times expensive, it lasts much longer. Instead of having replacement two to three times a year, it can be used for a number of years since the lighter composite crutch could sustain greater than 1100 pounds load. 
Table 2: Comparison of crutch weight and strength.

\begin{tabular}{lcc}
\hline Crutch design & Weight (lb) & Strength (lbs) \\
\hline Invacare & 2.3 & 630 \\
Ergonomics & 1 & 715 \\
Pareto design (A) & 0.9498 & 921 \\
Pareto design (B) & 1.0945 & 1107 \\
Nash equilibrium (C) & 0.9532 & 926 \\
Weight leader strength follower (D) & 0.9532 & 926 \\
Strength leader weight follower (E) & 0.9879 & 951 \\
Weight complete control (F) & 0.9499 & 922 \\
Strength complete control (G) & 1.0952 & 1100 \\
\hline
\end{tabular}

\section{Conclusion}

Collaborative design decisions involve designers from different discipline with different specific domain knowledge. The decision process is a sequence of phases or activities where mathematical modeling can employ. In this paper, a bi-level distributed framework based on Memetic Algorithm (MA) is proposed. Since the information communicated is neither the form of the decision function nor the decision space, private information is protected. In addition, in the cases where the information is not complete, the proposed framework can still guarantee the convergence to Pareto solutions. To demonstrate the applicability of the framework, a forearm crutch design is studied in details. The results confirm that converged Pareto set can be obtained for any form of decision function. While promising, the decision problem constructed is deterministic, our next step is to explore the use of this framework for design decisions under uncertainty. Computational efficient approach in the area of reliability-based design optimization would be explored.

\section{References}

[1] N. Alexandrov, "Editorial-multidisciplinary design optimization," Optimization and Engineering, vol. 6, no. 1, pp. 5-7, 2005.

[2] R. D. Braun and I. M. Kroo, "Development and application of the collaborative optimization architecture in a multidisciplinary design environment," in Multidisciplinary Design Optimization: State of the Art, pp. 98-116, SIAM, 1997.

[3] A. Badiru and V. Theodoracatos, "Analytical and integrative expert system model for design project management," Journal of Design and Manufacturing, vol. 4, pp. 195-213, 1995.

[4] A. E. Thal, A. Badiru, and R. Sawhney, "Distributed project management for new product development," International Journal of Electronic Business Management, vol. 5, no. 2, pp. 93-104, 2007.

[5] J. Sobieszczanski-Sobieski and R. T. Haftka, "Multidisciplinary aerospace design optimization: survey of recent developments," Structural Optimization, vol. 14, no. 1, pp. 1-23, 1997.

[6] N. M. Alexandrov and R. M. Lewis, "An analysis of some bilevel approaches to multidisciplinary design optimization," Tech. Rep., Institute for Computer Applications in Science and Engineering, NASA Langley Research Center, Hampton, Va, USA, June 2000.

[7] C. C. Huang, "A multi-agent approach to collaborative design of modular products," Concurrent Engineering: Research and Applications, vol. 12, no. 1, pp. 39-47, 2004.

[8] W. D. Li, S. K. Ong, J. Y. H. Fuh, Y. S. Wong, Y. Q. Lu, and A. Y. C. Nee, “Feature-based design in a distributed and collaborative environment," CAD Computer Aided Design, vol. 36, no. 9, pp. 775-797, 2004. 
[9] T. W. Simpson, C. C. Seepersad, and F. Mistree, "Balancing commonality and performance within the concurrent design of multiple products in a product family," Concurrent Engineering Research and Applications, vol. 9, no. 3, pp. 177-190, 2001.

[10] G. Hernandez, C. C. Seepersad, and F. Mistree, "Designing for maintenance: a game theoretic approach," Engineering Optimization, vol. 34, no. 6, pp. 561-577, 2002.

[11] H.-J. Choi, J. H. Panchal, J. K. Allen, D. Rosen, and F. Mistree, "Towards a standardized engineering framework for distributed, collaborative product realization," in Proceedings of the ASME Design Engineering Technical Conference, vol. 1, pp. 985-995, Chicago, Ill, USA, 2003.

[12] H. J. Wassenaar and W. Chen, "An approach to decision-based design with discrete choice analysis for demand modeling," Journal of Mechanical Design, vol. 125, no. 3, pp. 490-497, 2003.

[13] H. J. Wassenaar, W. Chen, J. Cheng, and A. Sudjianto, "Enhancing discrete choice demand modeling for decision-based design," Journal of Mechanical Design, vol. 127, no. 4, pp. 514-523, 2005.

[14] E. Nikolaidis, "Decision-based approach for reliability design," Journal of Mechanical Design, vol. 129, no. 5, pp. 466-475, 2007.

[15] H. Simon, "A behavioral model of rational choice," Quarterly Journal of Economics, vol. 6, pp. 99-118, 1955.

[16] K. Lewis and F. Mistree, "Collaborative, sequential, and isolated decisions in design," Journal of Mechanical Design, vol. 120, no. 4, pp. 643-652, 1998.

[17] K. Lewis and F. Mistree, "Foraging-directed Adaptive Linear Programming (FALP): a hybrid algorithm for discrete/continuous design problems," Engineering Optimization, vol. 32, no. 2, pp. 191217, 1999.

[18] M. G. Fernandez, J. H. Panchal, J. K. Allen, and F. Mistree, "An interactions protocol for collaborative decision making-concise interactions and effective management of shared design spaces," in Proceedings of the ASME Design Engineering Technical Conferences and Computer and Information in Engineering Conference, Long Beach, Calif, USA, 2005, Paper No. DETC2005-85381.

[19] S. Boyd, "EE392o Course Notes: Sub-Gradient Methods," Stanford University, Stanford, Calif, USA, 2004, http://www.stanford.edu/class/ee392o/.

[20] J. Sobieszczanski-Sobieski, "A linear decomposition method for large optimization problems blueprint for development," National Aeronautics and Space Administration, NASA/TM-832481982, 1982.

[21] J. Sobieszczanski-Sobieski, "Optimization by decomposition: a step from hierarchic to nonhierarchic systems," Tech. Rep. TM 101494, NASA, Hampton, Va, USA, September 1988.

[22] J. Sobieszczanski-Sobieski, B. B. James, and A. R. Dovi, "Structural optimization by multilevel decomposition," AIAA journal, vol. 23, no. 11, pp. 1775-1782, 1985.

[23] R. Balling and J. Sobieszczanski-Sobieski, "Optimization of coupled systems: a critical overview," in Proceedings of the 5th AIAA/NASA/USAF/ISSMO Symposium on Multidisciplinary Analysis and Optimization, Panama City Beach, Fla, USA, September 1994.

[24] J. Sobieszczanski-Sobieski and S. Kodiyalam, "BLISS/S: a new method for two-level structural optimization," Structural and Multidisciplinary Optimization, vol. 21, no. 1, pp. 1-13, 2001.

[25] G. M. Fernandez, W. D. Rosen, K. J. Allen, and F. Mistree, "A decision support framework for distributed collaborative design and manufacture," in Proceedings of the 9th AIAA/ISSMO Symposium on Multidisciplinary Analysis and Optimization, Atlanta, Ga, USA, Sepember 2002.

[26] D. Kumar, C. Hoyle, W. Chen, N. Wang, G. Gomez-levi, and F. Koppelman, "Incorporating customer preferences and market trends in vehicle packaging design," in Proceedings of the DETC, International Design Engineering Technical Conferences and Computers and Information in Engineering Conference (ASME '07), Las Vegas, NV, USA, Sepember 2007.

[27] B. Besharati, L. Luo, S. Azarm, and P. K. Kannan, "Multi-objective single product robust optimization: an integrated design and marketing approach," Journal of Mechanical Design, vol. 128, no. 4, pp. 884 892, 2006.

[28] C. -S. Shiau and J. Michalek, "A game-theoretic approach to finding market equilibria for automotive design under environmental regulation," in Proceedings of the ASME International Design Engineering Technical Conferences, vol. 6, pp. 187-195, Las Vegas, Nev, USA, September 2008.

[29] D. B. Sieger, A. B. Badiru, and M. Milatovic, "A metric for agility measurement in product development," IIE Transactions, vol. 32, no. 7, pp. 637-645, 2000. 


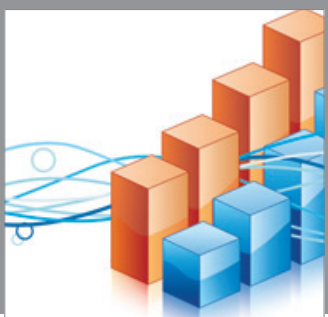

Advances in

Operations Research

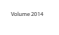

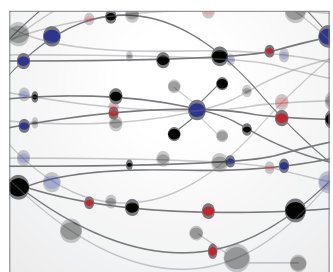

\section{The Scientific} World Journal
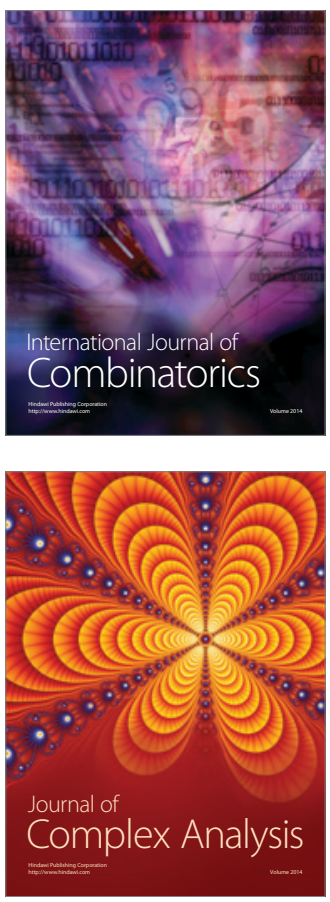

International Journal of

Mathematics and

Mathematical

Sciences
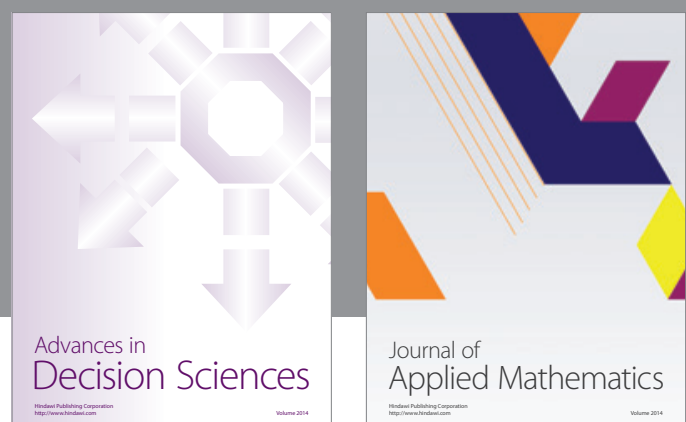

Journal of

Applied Mathematics
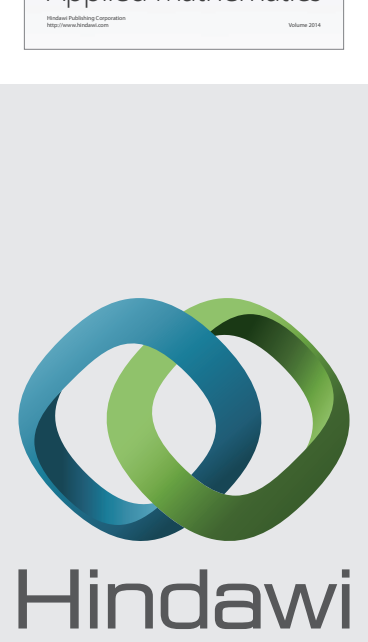

Submit your manuscripts at http://www.hindawi.com
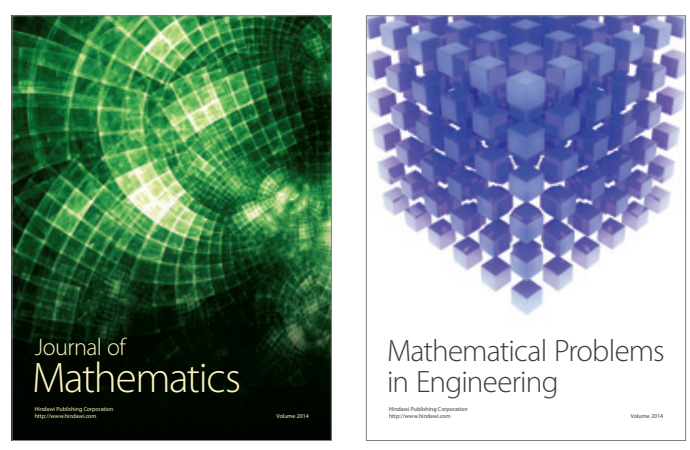

Mathematical Problems in Engineering
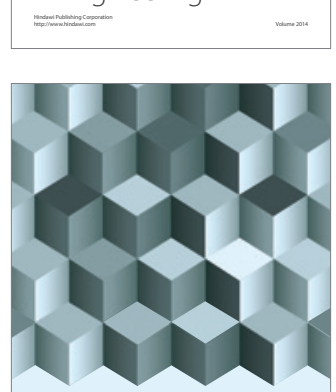

Journal of

Function Spaces
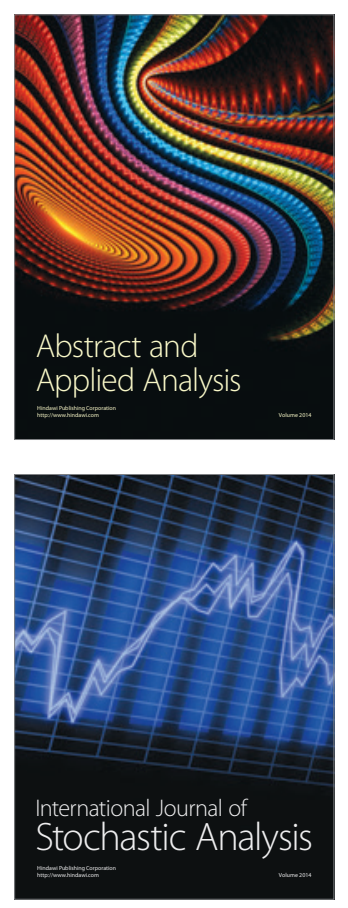

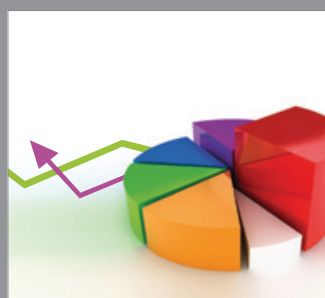

ournal of

Probability and Statistics

Promensencen
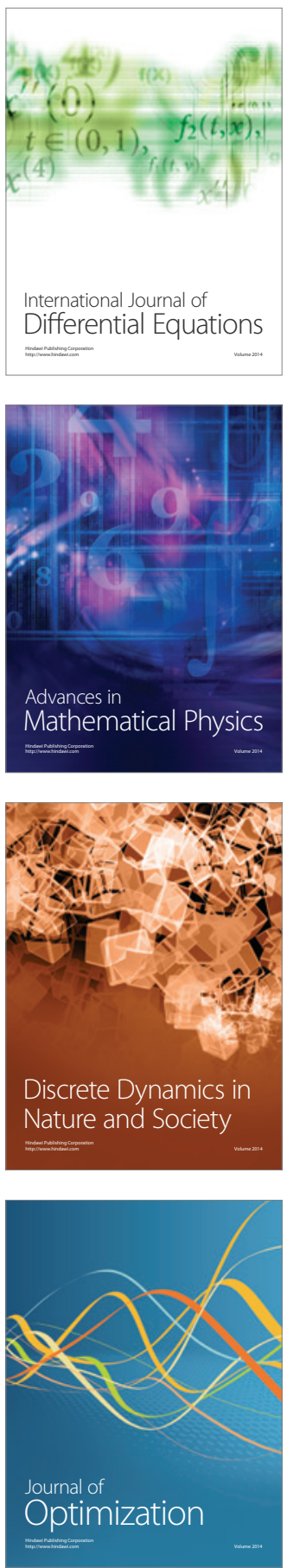